setting out the rationale of the collection.

That rationale appears to be centred on crystal structure and microstructure. The first chapter is a (necessarily) extremely superficial survey of crystallographic principles (including diffraction methods, Brillouin zones, and all sorts of goodies) in 45 pages, by $\mathrm{H}$. and S. G. Lipson. It is a valiant effort, but really it represents an attempt to compress a major textbook by a factor of ten, and was doomed to failure before it began. Crocker's chapter on crystal defects covers a more restricted field and is more digestible, but in places it lays itself open to the question "so what?". There is not much on the influence of defects on properties. Hull and Roberts deal very clearly with the formal crystallography of mechanical twinning, and particularly with the factors governing choice of twinning elements. There has been much work on these factors in recent years, and many references are quoted. Within its rather narrow terms of reference this is a valuable review. Willis and Williams deal rather briefly with defects in non-stoichiometric compounds, with special reference to uranium dioxide and neutron diffraction. This is extremely specialized matter and its somewhat dated nature is revealed by the lack of any reference to the recent use of channelled bombarding ions to locate interstitial solute atoms.

The book contains two heavyweight chapters which go a long way to justify purchase: Owen and Schoen on martensitic structures and especially Nicholson and Davies on development of microstructures. The former deals remarkably clearly with the conceptually very difficult crystallography of martensitic transformations, coupled with a discussion of the nucleation and growth of martensite. There is an invaluable bibliography. The chapter on microstructures is the only one of its kind known to myself, and covers thermodynamic principles, formation of both pure and polyphase solids from the melt, and the main categories of solidsolid transformation. Always the emphasis is on the geometry of microstructures and on the energetic considerations that determine them. This chapter will be most useful introductory reading for final year undergraduates in metallurgy and materials science--and for their teachers.

During the past ten years, a large number of surveys of particular fields of materials science have been published in smørgasbord volumes: many of the references at the ends of the chapters in the volume under review are to articles in other collections. ... Progress in This and Advances in That, the Relation Between or the Mechanism of Many invaluable surveys lie deeply hidden in volumes such as Progress in
Solid State Chemistry, where a physical metallurgist, say, would never think of browsing, or in Chemical and Mechanical Behaviour of Inorganic Materials (recently reviewed in these columns). It is time for an enterprising publisher to compile a periodic Good Food Guide to Scientific Smørgasbord, perhaps in each of several categories, say Materials Science, Solid State Physics, and so on. If such a compilation could incorporate a system of grades, as does the Consumers' Association's invaluable guide to British restaurants, so much the better. At the current price of dining out, indigestion is hard to bear.

R. W. CAHN

\section{Plasmas Everywhere}

Solid State Plasmas. By M. F. Hoyaux. (Applied Physics Series.) Pp. 159. (Pion: London, 1970.) $£ 2.50$ boards; $£ 1.80$ paper.

A LARGE number of moving charged particles having sufficiently high density may be defined as a plasma. Plasmas are usually considered in the liquid or gaseous phase but some of their properties are displayed by solids, more particularly conductors and semiconductors. It is the purpose of this monograph to outline the range of analogies which may be drawn, and the similarities which exist, between solid state and other plasmas. To do this, the author has written three interrelated reviews, each a condensed Report on Progress in Physics, emphasizing physical aspects rather than mathematical description.

An outline of solid state theory is followed by a summary of the relevant plasma physics and the two are fused in a final discussion of work on solid state plasmas. Professor Hoyaux writes with a sophistication which requires the reader to be familiar with both solid state and plasma theory if the full merit of his monograph is to be appreciated. It is a most interesting book.

An encouraging feature of physics generally and certainly of the topics outlined here is that apparent conflicts may be reconciled to the point where a common description is feasible. Both gaseous and solid state plasmas strongly resist any attempt at significant departure from charge neutrality. Both are capable of confinement by a magnetic bottle and are subject to instabilities. Collective behaviour is similar and common wave types may propagate in both media. In a gas, however, all charged particles are capable of playing an equal part while a solid state plasma is strongly degenerate with only a few electrons or holes at the Fermi surface responsible for transport phenomena.

Scattering by the solid lattice dominates its plasma properties and a wave mechanical description is necessary.
This introduces the concept of an anisotropic and varying effective mass which has no true equivalent in a gaseous plasma. Solid state plasmas have much higher densities, plasma frequencies and dielectric constants but a much lower temperature range than their gaseous counterparts.

So far the study of solid state plasmas has yielded further information on the Fermi surfaces of the solid; the future holds promise for the development of wider applications. JOHN PAIN

\section{Crystallography}

Fourier Methods in Crystallography. By G. N. Ramachandran and R. Srinivasan. (Wiley Monographs in Crystallography.) Pp. xiii +259 . (Wiley Interscience: New York and London, December 1970.) $£ 7.50$.

THIs monograph is the third of a series on various specialized aspects of crystallography. It is concerned with the problem of deriving a crystal structure by Fourier transformation of X-ray intensities, a subject with which the authors have been actively concerned for at least a decade.

The arrangement of material is very similar to that of Ramachandran's 1964 review of the same topic in Advanced Methods of Crystallography; new material has been added, however, and the literature coverage, which is an important part of a book of this kind, is particularly thorough. The authors begin by describing briefly the properties of Fourier transforms and go on to discuss the various ways they may be applied in diffraction studies. The Patterson function, and the imageseeking functions derived from it, are then dealt with, and the information contained in the phases illustrated by examples of phase and random syntheses. The main theme of the book is introduced by a detailed discussion of the various Fourier techniques which may be applied when part of the structure is known. Four chapters are devoted to a consideration of the best strategy to adopt with conventional heavy atom problems, and the improvement in resolution obtained by suitably weighting the Fourier coefficients is convincingly shown. The elaborations required for structure solution by the methods of isomorphous replacement and anomalous dispersion are then considered separately. Finally, Fourier techniques for applying both of these methods simultaneously are discussed.

The book is thus a compact and authoritative review of developments in structure solution by Fourier methods during the past fifteen years. It is the first survey of this topic for some time, and it should therefore prove useful to 